\title{
Positive and Negative Motor Signs of Head Motion in Cerebral Palsy: Assessment of Impairment and Task Performance
}

\author{
Miguel A. Velasco, Rafael Raya, Ramón Ceres, Alejandro Clemotte, \\ Antonio Ruiz Bedia, Teresa González Franco, and Eduardo Rocon
}

\begin{abstract}
This paper analyzes the presence of positive and negative motor signs in people with cerebral palsy (CP). Positive motor signs are those that lead to involuntarily increased frequency or magnitude of muscle activity. Negative motor signs describe insufficient muscle activity or insufficient control of muscle activity. In this paper, a head-mounted alternative computer interface based on inertial technology was used to assess motor signs in seven users with CP. Task performance and control of posture was related to the impairment. There are no significant differences between users with CP and healthy control participants in the frequency domain of the head movement. Results suggest that this kind of motor disorders is not related to positive motor signs. Moreover, a control mode based on posture more than on movements is not optimum; an alternative control mode must be specially designed for users with poor postural control.
\end{abstract}

Index Terms-Cerebral palsy (CP), head, human-computer interface, inertial, motor disorder.

\section{INTRODUCTION}

C EREBRAL Palsy (CP) is one of the most severe disabilities in childhood and makes heavy demands on health, educational, and social services, as well as on families and children themselves. The most widely and cited definition of CP states that it is "a disorder of movement and posture due to a defect or lesion of the immature brain" [1]. The complete definition also affirms that, "for practical purposes, it is usual to exclude from CP those disorders of posture and movement that are 1) of short duration; 2) due to progressive disease; or 3) due solely to mental deficiency" although most authors only cite the first brief sentence. The prevalence of $\mathrm{CP}$ is internationally $1.5-2.8$ cases per 1000 births. Only in the U.S., 0.5 million infants are affected by $\mathrm{CP}$ [2]. In Europe, these figures are even higher; the overall rate for the period from 1980 to 1990 was 2.08 per 1000 live births [3]. The work "Surveillance of Cerebral Palsy in Europe: a Collaboration of CP surveys and registers" presented a consensus that was reached on a definition of $\mathrm{CP}$, description and classification in terms of nosology, and topography and function (severity). The nosological classification divides CP into three types: spastic, ataxic, and dyskinetic. Spastic CP is characterized by at least two of these signs: abnormal pattern of posture and/or movement, increased tone, and pathological reflexes. It may be either bilateral or unilateral. Ataxic CP is characterized by both abnormal pattern of posture and/or movement and loss of orderly muscular coordination; movements are performed with abnormal force, rhythm, and accuracy. Dyskinetic CP is dominated by both abnormal pattern of posture and/or movement, and involuntary, uncontrolled, recurring, occasionally stereotyped movements [4].

\section{A. Positive and Negative Motor Signs}

Children with motor disorders often have a combination of multiple symptoms and clinical signs that contribute to their disability. One general classification of motor signs distinguishes two basic categories: positive signs and negative signs [5]. Positive motor signs can be defined as those that lead to involuntarily increased frequency or magnitude of muscle activity, movement, or movement patterns. Examples include hypertonia, chorea, tics, and tremor. Low-frequency involuntary movements such as athetosis are not related to positive motor signs. Negative motor signs describe insufficient muscle activity or insufficient control of muscle activity. Examples include weakness, impaired selective motor control, ataxia, and apraxia [6]. Positive motor signs are often easier to detect in the clinic, and there has been significant effort to identify and quantify such signs. Negative motor signs are often more difficult to quantify, and there are fewer effective treatments. Positive and negative motor signs are often simultaneously present and may be linked rather than independent features of a motor disorder [7].

These definitions are useful in order to facilitate the development of rating scales to assess improvement or deterioration with time [7]. Furthermore, efficiency of physical, cognitive, and functional therapies can be improved if they adapt to the specific needs of the users.

\section{B. Aim of This Paper}

The initial hypothesis for this paper is that negative motor signs are predominant in people affected by CP. It has been said that positive motor signs can be described by increments in the 


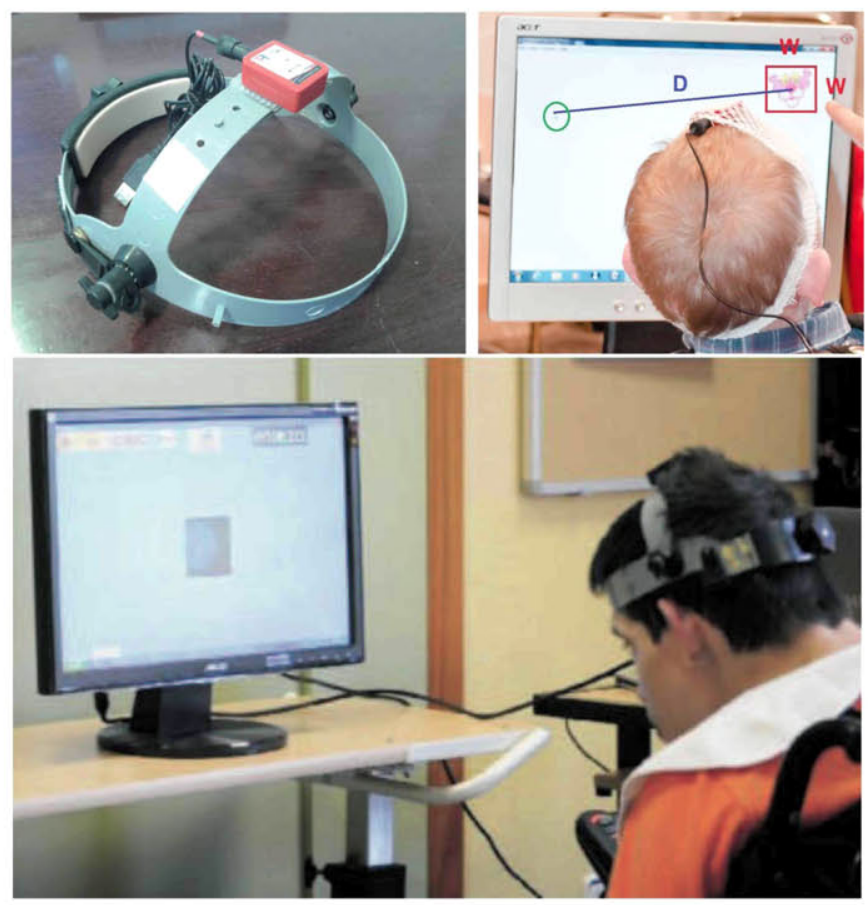

Fig. 1. ENLAZA interface: IMU and software. Upper left corner: IMU attached to the helmet. Upper right and bottom: participants from the CP group during one of the work sessions. The target is a squared figure with size $W \times W$ pixels located at a distance $\mathrm{D}$ from the cursor.

frequency of muscle activity. This means that frequency components well above the dominant frequency (DF) of voluntary movements $(1-2 \mathrm{~Hz})$ will be found in involuntary movements if positive motor signs are identified (e.g., tremor is characterized by frequencies around $5-7 \mathrm{~Hz}$ ). Head motion in users with $\mathrm{CP}$ and healthy subjects (HSs) will be analyzed in the frequency domain. No significant difference between groups might be an indicator of the absence of positive motor signs responsible for motor disorders.

On the contrary, we expect to find significant differences in the performance of the task and head range of motion (ROM) as a direct consequence of the motor and postural disorder, described by negative motor signs.

\section{Methodology}

The methodology is based on a reaching task. Eye and face tracking interfaces are powerful pointing devices for people with motor disorders and a very natural form of pointing as people tend to look at the object they wish to interact with. However, severe disability caused by $\mathrm{CP}$ requires a different approach to reduce the effect of involuntary movements on human-machine interaction. Users wore a hat or helmet with an inertial sensor attached to it (see Fig. 1). This alternative interface, called ENLAZA, allowed them to control the cursor of the computer with movements of their heads. Users were instructed to locate the mouse pointer over a static target as quickly as possible. All participants had experience with the interface; therefore, only a short training for this particular task was needed. Each work session consisted of reaching 17 targets on the screen, one for practicing and 16 for assessment.
TABLE I

USER NOSOLOGICAL, TOPOGRAPHICAL, AND FUnCTIONAL CAPACITY Classification

\begin{tabular}{cccc}
\hline User & Nosology & Topography & Function \\
\hline CP1 & Spastic & Quadriplegia & Severe \\
CP2 & Dystonic-Athetoid & Quadriplegia & Severe \\
CP3 & Dystonic-Athetoid & Quadriplegia & Severe \\
CP4 & Dyskinetic & Quadriplegia & Severe \\
CP5 & Dyskinetic & Quadriplegia & Severe \\
CP6 & Spastic & Quadriplegia & Severe \\
CP7 & Mixed & Diplegia & Severe \\
\hline
\end{tabular}

TABLE II

USER DESCRIPTION: RELEVANT CHARACTERISTICS

\begin{tabular}{|c|c|c|c|}
\hline User & Tone & Associated Movements & Intellectual ability \\
\hline CP1 & Hypertonia & $\begin{array}{l}\text { No movements } \\
\text { associated }\end{array}$ & Normal \\
\hline $\mathrm{CP} 2$ & Hypertonia & Athetoid movements & Normal \\
\hline $\mathrm{CP} 3$ & Dystonia & Ballistic movements & Normal \\
\hline CP4 & Hypotonia & Dystonic movements & Normal \\
\hline CP5 & Hypotonia & $\begin{array}{l}\text { No movements } \\
\text { associated }\end{array}$ & $\begin{array}{l}\text { Mild intellectual } \\
\text { disability }\end{array}$ \\
\hline CP6 & Hypertonia & Athetoid movements & $\begin{array}{l}\text { Medium intellectual } \\
\text { disability }\end{array}$ \\
\hline CP7 & Hypotonia & $\begin{array}{l}\text { No movements } \\
\text { associated }\end{array}$ & $\begin{array}{l}\text { Medium intellectual } \\
\text { disability }\end{array}$ \\
\hline
\end{tabular}

The difficulty of the task, that depended on the distance and size of the targets, was the same for all users. Two values of distance and target size were chosen; the target was located at a distance of 300 or 500 pixels from the position of the cursor. Target's size was $100 \times 100$ or $200 \times 200$ pixels large. Hence, there were four combinations of size and distance of the targets. In a session, the user had to perform four repetitions in a randomized order of those four distance-size combinations, for a total of 16 . Screen resolution was $1366 \times 768$ pixels.

\section{A. Participants}

Eleven subjects participated in the study (age $31.8+/$ -9.2). Prior to the beginning of the tests, they had completed $21+/-7$ sessions in two months. Three of the participants left the study after a small number of sessions. Two of them had very poor motor control and presented difficulties to complete the task. Both continued using ENLAZA in less challenging activities. The third one was first included in the study, but he was dropped out because he did not fully understand the proposed task due to his intellectual disability. Another participant had good performance but was not able to complete some of the sessions in time. Their tests are not included in the analysis. For the control group, three volunteers participated in the experiments (age $30+/-2.5$ ). They completed $3+/-1$ training sessions before starting the study. Tests took place at ASPACE Cantabria (Santander, Spain), a center specialized in $\mathrm{CP}$ and similar disorders. The control group or HSs participated in the tests at the Bioengineering Group of the Spanish National Research Council (Madrid, Spain). Table I depicts user classification. Some other descriptors considered relevant for the study can be observed in Table II. 


\section{B. Inertial Interface and Assessment Software}

The inertial interface consists of a headset with a cap and an inertial measurement unit (IMU). The IMU was developed in the Bioengineering Group of the Spanish National Research Council in collaboration with Technaid, Ltd. It integrates a three-axis gyroscope, an accelerometer, and a magnetometer. It uses Coriolis force principle to measure angular velocity and Hooke's law for acceleration. The magnetometer measures Earth's magnetic field. The IMU design is based on microelectromechanical system technology and is available in a small package $(27 \times 35 \times 13 \mathrm{~mm}, 27 \mathrm{~g})$. It is able to measure $+/-2 \mathrm{G},+/-3 \mathrm{~g}$, and $+/-500^{\circ} / \mathrm{s}$ in the three axes. The angular resolution of the device is $0.05^{\circ}$, static accuracy less than $1^{\circ}$, and dynamic accuracy of about $2^{\circ}$ RMS.

IMU orientation is estimated based upon the data recorded by the accelerometer, gyroscope, and magnetometer. The three Euler angles $\alpha, \beta$, and $\gamma$ (in the frontal, sagittal, and transverse planes) are calculated from the rotation matrix

$$
\begin{aligned}
\boldsymbol{R}_{G S} & =\boldsymbol{R}_{S} \cdot\left(\boldsymbol{R}_{G}\right)^{-1} \\
\alpha & =a \tan \left(-\frac{\boldsymbol{R}_{G S(2,3)}}{\boldsymbol{R}_{G S(3,3)}}\right) \\
\beta & =a \sin \left(\boldsymbol{R}_{G S(1,3)}\right) \\
\gamma & =a \tan \left(-\frac{\boldsymbol{R}_{G S(1,2)}}{\boldsymbol{R}_{G S(1,1)}}\right)
\end{aligned}
$$

where $\boldsymbol{R}_{G}$ is defined as the rotation matrix of the global reference system corresponding to the neutral position of the head (looking at the center of the screen), and $\boldsymbol{R}_{S}$ as the rotation matrix that describes the orientation of the sensor at each frame.

For the purpose of this paper, the mouse pointer is controlled with an absolute system, meaning that there is a unique relationship between head orientation and location of the pointer and that, after a calibration process, all pixels in the screen are reachable for the user's head ROM. During the calibration, a therapist adjusts the gain of the transfer function that translates the orientation of the head into a location of the pointer on the screen. The software captures data used to assess the following.

1) Impairment. The device captures kinematic parameters such as acceleration, angular velocity, or ROM, which is correlated with normal and abnormal patterns (physical impairment).

2) Performance in the task. The application captures the positions of the mouse pointer and target during the session.

\section{Assessment of Impairment}

Two metrics for the quantification of positive and negative motor signs were proposed in previous studies: frequency of movement and ROM of user's head [8], [23]. ROM is defined as the difference between the maximum and minimum Euler angles measured in one of the anatomical planes: frontal, sagittal, or transverse (Euler angles $\alpha, \beta$, and $\gamma$ ). The presence of

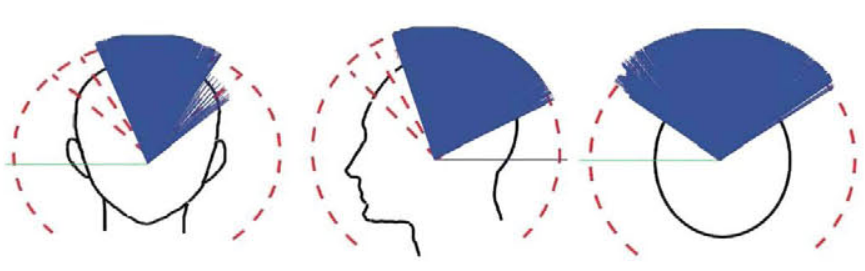

Fig. 2. Representation of the head orientation in the frontal, sagittal, and transverse planes for one user with $\mathrm{CP}$. Recordings correspond to a total of 16 reaching tasks with the ENLAZA interface. The Euler angles displayed are, from left to right, $\alpha, \beta$, and $\gamma$.
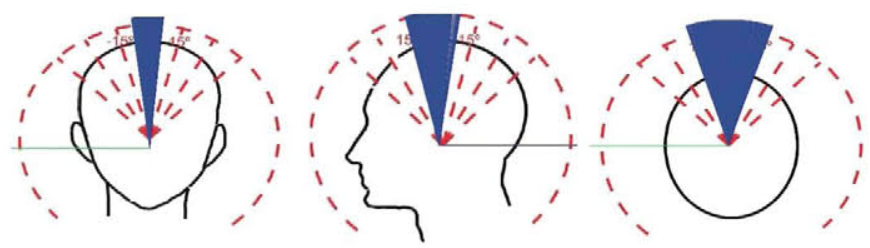

Fig. 3. Representation of the head orientation in the frontal, sagittal, and transverse planes for a HS. Recordings correspond to a total of 16 reaching tasks with the ENLAZA interface. The Euler angles displayed are, from left to right, $\alpha, \beta$, and $\gamma$.

positive motor signs in the involuntary movements of users can be assessed by analyzing the frequency of those movements. Thus, the power spectrum estimation of the signals recorded by the gyroscopes in the three axes will be calculated. Posture disorders, related to negative motor signs, can be studied by analyzing the ROM for the three planes, i.e., frontal, sagittal, and transverse. Figs. 2 and 3 depict the three angles measured in one user of each study group.

The presence of positive or negative motor signs has implications for the design of the inertial interface. If positive motor signs (increased frequency) are identified and involuntary movements are related to higher spectral components, those frequencies could be digitally filtered. On the contrary, if negative motor signs (related to poor postural control) are detected, a different approach based on movement rather than orientation is needed.

\section{Assessment of Task Performance}

In addition to the analysis of impairment, we propose two parameters for the assessment of task performance. Throughput, defined by the standard "ISO 9241-Part 9. Requirements for non-keyboard input devices", is a parameter used to measure the performance in a reaching task. It is based on the time needed by the user to complete the task but also takes into account the difficulty of the proposed task, and it somehow normalizes the time estimation. Thus, throughput is considered a more robust parameter than reaching time itself. The difficulty of the task is quantified by the index of difficulty (ID), which is based on the size of the target $W$, and the initial distance from the mouse pointer to the target $D$ (see Fig. 1). ID can be calculated as

$$
\mathrm{ID}=\log _{2}\left(\frac{D}{W}+1\right) .
$$

The throughput during a single task is defined as the division between the ID and the reaching time, and its units are bits per 
second [9], [14]. It is widely accepted as a tool for the quantitative evaluation of pointing devices for general population [15][18] and people with spinal cord injury [19], [20] or CP [21][23]. Authors in those studies presented values of throughput in healthy users of $2.24+/-0.88 \mathrm{bits} / \mathrm{s}$ for alternative pointing devices, whereas the throughput for traditional computer mice is usually around $3.5-4.5$ bits/s.

In addition to the identification of postural disorders, ROM can be used for the analysis of task performance. Measured $\mathrm{ROM}, \mathrm{ROM}_{M}$, is defined as the ROM calculated during a reaching task. A required ROM, $\mathrm{ROM}_{R}$, could be estimated based on the distance between the mouse pointer and the target at the beginning of the task and the transfer function for the head-pointer movement (degrees needed to move the pointer one pixel in the screen). The ratio of ROMs, $\mathrm{ROM}_{\text {ratio }}$, is defined as the division of $\mathrm{ROM}_{M}$ and $\mathrm{ROM}_{R}$ and can be a descriptor of how precise user's movements are, i.e.,

$$
\mathrm{ROM}_{\text {ratio }}=\frac{\mathrm{ROM}_{M}}{\mathrm{ROM}_{R}} .
$$

The alternative interface allows users to choose whether they control the mouse pointer in the horizontal plane with head movements in transverse or frontal plane (defined as "normal" or "lateral" control). The maximum ROM reachable by the head is larger in the transverse plane than in the frontal plane; hence, absolute values of ROM cannot be compared unless all users work with the same type of control. The new ratio of ROMs presented in this paper is independent of the chosen type of control because differences between controls are reflected in the value of $\mathrm{ROM}_{R}$. A value of $\mathrm{ROM}_{\text {ratio }}$ close to the unit would mean that the movement is efficient; therefore, no overreaching was detected in the reaching task, and the user did not need several submovements but only a single movement in order to complete the task. We expect to measure higher values of $\mathrm{ROM}_{\text {ratio }}$ in users with $\mathrm{CP}$ as a consequence of poor control of motion and posture.

\section{E. Comparison of Parameters for $\mathrm{CP}$ and Control Groups}

A Lilliefors normality test was run for the nine calculated parameters. Results concluded $(p<0.05)$ that the hypothesis of normality could be rejected in a number of them. Thus, a parametric test could not be used for the comparison of the populations. A nonparametric method was used instead.

The Wilcoxon signed-rank test was used to assess whether the measured parameters for the control and the CP group differed. Our hypothesis is that the throughput and the ratio of ROMs would be significantly different for the two groups. On the other hand, the presence of negative motor signs would be reflected in none statistical differences between the frequencies measured for the healthy volunteers and the subjects with $\mathrm{CP}$. The null hypothesis $H_{0}$ is rejected with $p<0.05$ and states that both populations are equal in terms of median.

\section{RESULTS}

The performance during the task was higher in the control group. Median values of the throughput were 0.57 and

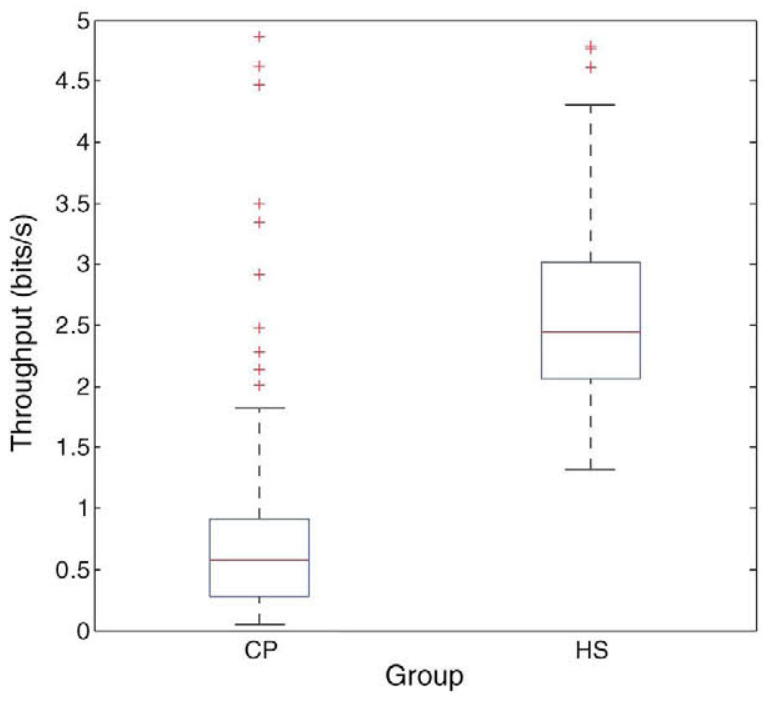

Fig. 4. Measure of throughput for the two groups. The box plots represent the values measured for each task during the work sessions of CP and HSs.
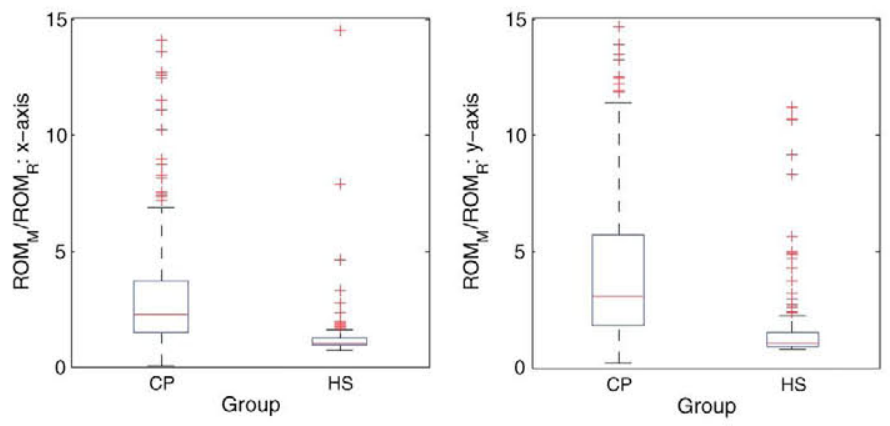

Fig. 5. Box plots representing measured ROM versus required ROM for the two groups: CP and HSs. Left: $x$-axis. Right: $y$-axis.

2.44 bits/s in the CP and control groups. Differences in the interquartile ranges (IQRs) were smaller: 0.63 and $0.95 \mathrm{bits} / \mathrm{s}$ for $\mathrm{CP}$ and $\mathrm{HS}$ groups due to the existing homogeneity of performance within the groups (see Fig. 4). The ratio of $\mathrm{ROM}_{M}$ and $\mathrm{ROM}_{R}$ is represented in Fig. 5. As expected, it was very close to the unit in HSs. Medians calculated were 1.01 and 1.06 in the $x$-axis and $y$-axis, respectively. In people with $\mathrm{CP}$, those values were 2.22 and 3.08 . This increase (38\%) in the measured ROM in the $y$-axis is consistent with the poorer postural control in the frontal and sagittal planes identified in users with cervical hypotonia. The IQR for both axes is around 7-8 times larger in the CP group, due to the heterogeneity of the user's tone and control of posture. The frequency analysis of the head motion in both groups displayed very low frequency components in the range between 0.5 and $2.5 \mathrm{~Hz}$. As shown in Fig. 6, 75\% of the spectral components were below $3.5 \mathrm{~Hz}$. Increased frequency cannot be observed in the recorded movements.

The 25th and 75th quartiles, as well as the median value of throughput (in bits per second), $\mathrm{ROM}_{\text {ratio }}$, dominant frequency, and bandwidth (in hertz) calculated for the two population groups, can be found in Table III.

The statistical analysis determined that not all the measured parameters fitted a normal distribution (see Table IV). The lowest $p$-values estimated in the Lilliefors test corresponded to the parameters used to quantify task performance: throughput 

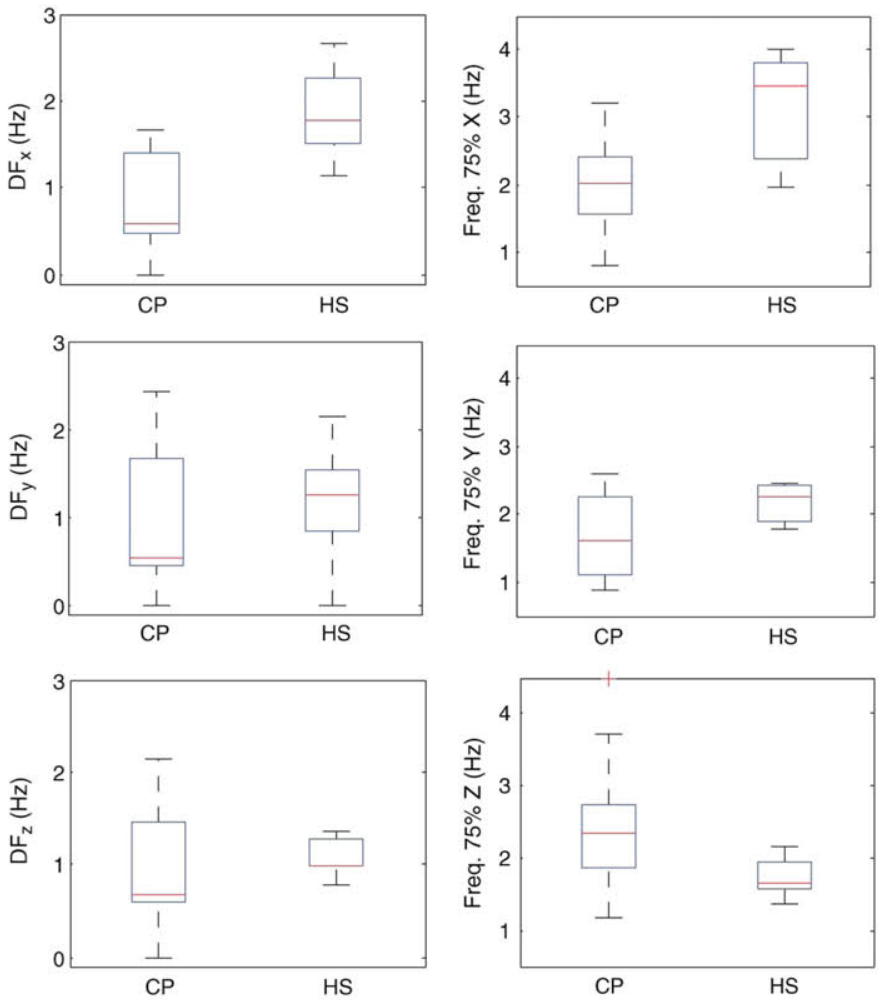

Fig. 6. (First row) DF for both groups in the three rotations: roll, pitch, and yaw. (Second row) Bandwidth at $75 \%$ of the total energy of the signal.

TABLE III

Distribution OF PARAMETERS

\begin{tabular}{lllllllll}
\hline & \multicolumn{2}{c}{ 25th Q. } & & \multicolumn{2}{c}{ Median } & & \multicolumn{2}{c}{ 75th Q. } \\
\cline { 2 - 3 } Parameter & CP & HS & & CP & HS & & CP & HS \\
\hline Throughput & 0.28 & 2.06 & & 0.57 & 2.44 & & 0.91 & 3.01 \\
ROM $_{\text {ratio }_{x}}$ & 1.49 & 0.96 & & 2.24 & 1.01 & & 3.70 & 1.22 \\
ROM $_{\text {ratio }}$ & 1.80 & 0.95 & & 3.08 & 1.06 & & 5.73 & 1.50 \\
DF $_{x}$ & 0.49 & 1.52 & & 0.58 & 1.77 & & 1.40 & 2.27 \\
DF $_{y}$ & 0.45 & 0.84 & & 0.54 & 1.26 & & 1.66 & 1.54 \\
DF $_{z}$ & 0.58 & 0.98 & & 0.68 & 0.98 & & 1.46 & 1.27 \\
$75 \%$ Freq $_{x}$ & 1.57 & 2.38 & & 2.03 & 3.47 & & 2.40 & 3.81 \\
$75 \%$ Freq $_{y}$ & 1.10 & 1.90 & & 1.60 & 2.26 & & 2.26 & 2.41 \\
$75 \%$ Freq $_{z}$ & 1.85 & 1.56 & & 2.34 & 1.66 & & 2.73 & 1.95 \\
\hline
\end{tabular}

and ratio of ROMs. This leads to the use of a nonparametric test for the comparison of medians, such as the Wilcoxon signed rank test (see Table V). The null hypothesis $H_{0}$ in this test is that the median difference between pairs of observations is zero. Statistical differences were found in the throughput and ROM ratios for vertical and horizontal motion of the cursor. No significant differences were found in the frequency parameters. Thus, $H_{0}$ can be only rejected $(p<0.05)$ for throughput, $\mathrm{ROM}_{\text {ratio }_{x}}$ and $\mathrm{ROM}_{\text {ratio }_{y}}$.

\section{CONCLUSION}

There are some inherent limitations to the population under study and the experiment itself that must be taken into account in order to analyze the results. The disability of the sample in the $\mathrm{CP}$ group is rather heterogeneous in terms of tone, involuntary movement, and intellectual ability. To gather a
TABLE IV

RESULTS OF THE LILLIEFORS NORMALITY TEST

\begin{tabular}{lrrrrr}
\hline & \multicolumn{2}{c}{$\mathrm{CP}$} & & \multicolumn{2}{c}{$\mathrm{HS}$} \\
\cline { 2 - 3 } \cline { 5 - 6 } Parameter & $\mathrm{H}$ & p-value & & $\mathrm{H}$ & p-value \\
\hline Throughput & 1 & $<\mathbf{0 . 0 1}$ & & 1 & $\mathbf{0 . 0 2}$ \\
ROM $_{\text {ratio }}$ & 1 & $<\mathbf{0 . 0 1}$ & & 1 & $<\mathbf{0 . 0 1}$ \\
ROM $_{\text {ratio }}$ & 1 & $<\mathbf{0 . 0 1}$ & & 1 & $<\mathbf{0 . 0 1}$ \\
F $_{x}$ & 1 & $\mathbf{0 . 0 2}$ & & 0 & 0.50 \\
DF $_{y}$ & 1 & $<\mathbf{0 . 0 1}$ & & 0 & 0.50 \\
DF $_{z}$ & 1 & $\mathbf{0 . 0 1}$ & & 1 & $\mathbf{0 . 0 3}$ \\
$75 \%$ Freq $_{x}$ & 0 & 0.50 & & 0 & 0.23 \\
$75 \%$ Freq $_{y}$ & 0 & 0.31 & & 0 & 0.28 \\
$75 \%$ Freq $_{z}$ & 1 & $\mathbf{0 . 0 1}$ & & 0 & 0.19 \\
\hline
\end{tabular}

TABLE V

REsults of the WilcoXon Signed Rank Test. DIFFERENCES BETWEEN GROUPS

\begin{tabular}{ccr}
\hline Parameter & $\mathrm{H}$ & p-value \\
\hline Throughput & 1 & $<\mathbf{0 . 0 1}$ \\
ROM $_{\text {ratiox }}$ & 1 & $<\mathbf{0 . 0 1}$ \\
ROM $_{\text {ratio }}$ & 1 & $<\mathbf{0 . 0 1}$ \\
F $_{x}$ & 0 & 0.25 \\
$D F_{y}$ & 0 & 0.12 \\
FF $_{z}$ & 0 & 0.62 \\
$75 \%$ Freq $_{x}$ & 0 & 0.75 \\
$75 \%$ Freq $_{y}$ & 0 & 0.50 \\
$75 \%$ Freq $_{z}$ & 0 & 0.12 \\
\hline
\end{tabular}

larger CP group would be desirable for more robust statistical significance. In addition to this, some aspects, such as motivation or fatigue, were not quantified, although they may play an important role in the performance of the task.

The statistical analysis showed significant differences in parameters of task performance between the control group and the $\mathrm{CP}$ group. Throughput was significantly lower in people with $\mathrm{CP}$, whereas the ratio of measured versus required $\mathrm{ROM}$ was substantially higher in the $\mathrm{CP}$ group. These are the consequences of the poorer postural control of users with $\mathrm{CP}$ and the resulting lower performance in the reaching task.

The frequency analysis, however, produced different results. The median comparison test could not reject the null hypothesis, although some tendencies can be observed in Fig. 6. Given the earlier enumerated limitations, statistical analysis showed no significant differences between the movements of the CP and control groups in the frequency domain. The absence of increased frequency and the presence of increased ROM (due mostly to muscle weakness) are consistent with the predominance of negative motor signs.

Results also suggest that absolute control might not be the optimum control mode because it is based on posture more than on movements. An alternative is the relative control. It is based on the angular velocity measured by the gyroscopes. Its main advantage for users with decreased tone is that, even if the user is leaning forward or backward due to muscle weakness, he or she will still be able to move the mouse pointer with small head movements.

In future studies, relative control will be tested in users with cervical hypotonia and will be compared with absolute control in terms of performance. Preliminary results in ongoing 
experiments seem to indicate that relative control is indeed a better choice for these users, but a larger sample is needed in order to confirm the hypothesis.

\section{ACKNOWLEDGMENT}

The authors would like to thank ASPACE Cantabria, a center specialized in CP and similar disorders.

\section{REFERENCES}

[1] M. C. O. Bax, "Terminology and classification of cerebral palsy," Develop. Med. Child Neurol., vol. 6, no. 3, pp. 295-307, Jun. 1964.

[2] S. Winter, A. Autry, and M. Yeargin-Allsopp, "Trends in the prevalence of cerebral palsy in a population-based study," Pediatrics, vol. 110, no. 6, pp. 1220-1225, Dec. 2002.

[3] A. Johnson, "Prevalence and characteristics of children with cerebral palsy in Europe," Develop. Med. Child Neurol., vol. 44, no. 9, pp. 633640 , Sep. 2002.

[4] C. Cans, "Surveillance of cerebral palsy in Europe: A collaboration of cerebral palsy surveys and registers," Develop. Med. Child Neurol., vol. 42 , no. 12 , pp. $816-824$, Dec. 2000

[5] W. Z. Rymer and R. T. Katz, "Mechanisms of spastic hypertonia," Archives Phys. Med. Rehab., vol. 70, no. 2, pp. 144-155, Feb. 1989.

[6] M. F. Levin and A. G. Feldman, "The role of stretch reflex threshold regulation in normal and impaired motor control," Brain Res., vol. 657, no. 1/2, pp. 23-30, Sep. 1994

[7] T. D. Sanger et al., "Definition and classification of negative motor signs in childhood," Pediatrics, vol. 118, no. 5, pp. 2159-2167, Nov. 2006.

[8] R. Raya et al., "Positive and negative motor signs of head motion in cerebral palsy an analysis based on a wearable inertial human computer interface," in Proc. ISSNIP BRC, 2013, pp. 1-5.

[9] P. M. Fitts, "The information capacity of the human motor system in controlling the amplitude of movement," J. Exp. Psychol., vol. 47, no. 6 , pp. 381-391, Jun. 1954.

[10] S. K. Card, W. K. English, and B. J. Burr, "Evaluation of mouse, rarecontrolled isometric joystick, step keys, text keys for text selection on a CRT," Ergonomics, vol. 21, no. 8, pp. 601-613, 1978.

[11] J. O. Wobbrock, E. Cutrell, S. Harada, and I. S. Mackenzie, "An error model for pointing based on Fitts â law," in Proc. SIGCHI Conf. Human Factors Comput. Syst., 2008, pp. 1613-1622.

[12] J. O. Wobbrock, K. Shinohara, and A. Jansen, "The effects of task dimensionality, endpoint deviation, throughput calculation, experiment design on pointing measures and models," in Proc. SIGCHI Conf. Human Factors Comput. Syst., 2011, pp. 1639-1648.

[13] J. O. Wobbrock, A. Jansen, and K. Shinohara, "Modeling and predicting pointing errors in two dimensions," in Proc. SIGCHI Conf. Human Factors Comput. Syst., 2011, pp. 1653-1656.

[14] R. Soukoreff and I. S. Mackenzie, "Towards a standard for pointing device evaluation, perspectives on 27 years of Fitts' law research in HCI," Int. J. Human-Comput. Studies, vol. 61, no. 6, pp. 751-789, Dec. 2004.

[15] D. Natapov, S. J. Castellucci, and I. S. MacKenzie, "ISO 9241-9 evaluation of video game controllers," in Proc. Graph. Interface, 2009, pp. $223-230$.

[16] J. Musić, M. Cecić, and M. Bonković, "Testing inertial sensor performance as hands-free human-computer interface," WSEAS Trans. Comput., vol. 8, no. 4, pp. 715-724, Apr. 2009

[17] Y.S. Ryu, D. H. Koh, D. Ryu, and D. Um, "Usability evaluation of touchless mouse based on infrared proximity sensing," J. Usability Studies, vol. 7, no. 1, pp. 31-39, Nov. 2011.

[18] K. L. Norman and K. D. Norman, "Comparison of relative versus absolute pointing devices,” Digital Repository, Univ. Maryland, College Park, MD, USA, Tech. Rep. HCIL-2010-25, 2005.

[19] C. Choi et al., "An SEMG computer interface using three myoelectric sites for proportional two-dimensional cursor motion control and clicking for individuals with spinal cord injuries," Med. Eng. Phys., vol. 35, no. 6, pp. 777-783, Jun. 2013.

[20] C. A. M. Pereira, R. B. Neto, A. C. Reynaldo, M. C. M. Luzo, and R. P. Oliveira, "Development and evaluation of a head-controlled humancomputer interface with mouse-like functions for physically disabled users," Clinics, vol. 64, no. 10, pp. 975-981, Oct. 2009.

[21] R. S. Rao, R. Saliktar, and T. Rahman, "Evaluation of an isometric and a position joystick in a target acquisition task for individuals with cerebral palsy," IEEE Trans. Rehab. Technol., vol. 8, no. 1, pp. 118-125, Mar. 2000
[22] R. G. Radwin, G. C. Vanderheiden, and M. L. Lin, "A method for evaluating head-controlled computer input devices using Fitts' law," Human Factors, vol. 32, no. 4, pp. 237-251, Aug. 2003.

[23] A. Clemotte, R. Raya, R. Ceres, and E. Rocon, "Preliminary result from a multimodal interface for cerebral palsy users based on eye tracking and inertial technology," in Converging Clinical and Engineering Research on Neurorehabilitation, Biosystems \& Biorobotics, vol. 1, J. L. Pons, D. Torricelli, and M. Pajaro, Eds. Berlin, Germany: Springer-Verlag, 2013, pp. $443-448$

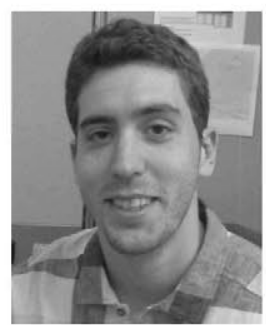

Miguel A. Velasco received the Telecommunication Engineering degree from Universidad de Zaragoza, Zaragoza, Spain, in 2009 and the Master's degree in biomedical engineering, with specialization in bioelectronics and biomedical instrumentation, from Universitat Politècnica de València, Valencia, Spain, in 2012. He is currently working toward the Ph.D. degree at the Bioengineering Group, Spanish National Council for Science Research, Madrid, Spain, working on assistive devices and alternative computer access systems for people with motor disorders. In 2012, he was with the Interuniversity Institute of Bioengineering Research and Technology Oriented to the Human Being (i3BH), Valencia, as a Research Associate focused on biosignal acquisition and processing.

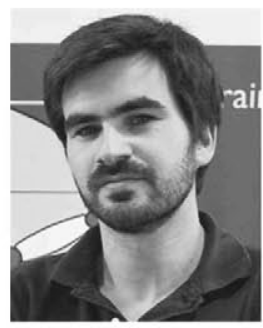

Rafael Raya received the Electronic and Automatic Engineering degree from the University of Córdoba, Córdoba, Spain, in 2006 and the M.S. and Ph.D. degrees from the University of Alcalá, Madrid, Spain, in 2008 and 2011, respectively.

He was a Postdoctoral Fellow with the Harvard Medical School, Boston, MA, USA, and with MOVE Institute (Vrije Amsterdam Universiteit, Amsterdam, The Netherlands). He is the author or coauthor of more than 40 publications, including international journals and conferences, and is a Reviewer of several international journals. He has actively participated in a number of national, European, and international research and technological development projects. His research activity is focused on assistive devices for people with cerebral palsy.

Dr. Raya is currently a Technical Coordinator with the Iberoamerican Association for Assistive Technology (AITADIS). He received the 2011 Best Spanish $\mathrm{Ph} . \mathrm{D}$. Thesis in Robotics from the Spanish Committee for Automation and the TR35 Award from Massachusetts Institute of Technology's journal, Technology Review (2013).

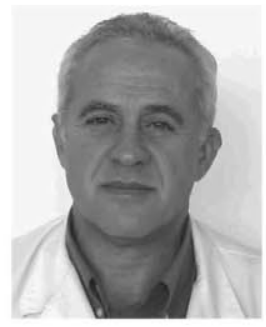

Ramón Ceres received the Ph.D. degree from the Complutense University of Madrid, Madrid, Spain.

He is currently a Professor of research with the Bioengineering Group, Spanish National Council for Science Research, Madrid. He has lead numerous national and international projects on research and development, contributing to the creation of two spin-off companies and other transfer technology processes. He is the author of more than 250 publications centered on the field of research in assistive technologies, particularly on sensors, interfaces, and

signal processing.

Dr. Ceres is the Founder President of the Latin American Association for Assistive Technologies (AITADIS). 


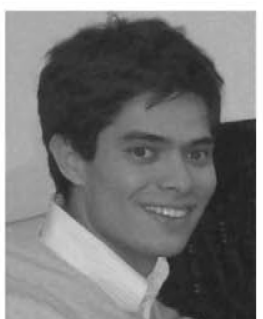

Alejandro Clemotte was born in Paraguay. He graduated with honors in the Universidad Católica Nuestra Señora de la Asunción, Asunción, Paraguay, in 2011. He graduated with honors in 2013, presenting a study about the ability of gaze tracking systems used by people with cerebral palsy. He received the Master's degree in engineering of electronic systems from the Polytechnic University of Madrid, Madrid, Spain. He is currently working toward the Ph.D. degree with the Bioengineering Group, Spanish National Council for Science Research (CSIC), Madrid. For his thesis, he is studying alternative computer access tools for people with cerebral palsy, with special emphasis on gaze tracking systems and its impact in the cognitive level of the users.

Antonio Ruiz Bedia received the M.Sc. degree in psychology.

$\mathrm{He}$ is currently an Associate Professor with Universidad de Cantabria, Santander, Spain, where he gives lectures in the module of "Education of children with cerebral palsy" of the Ed.S. degree in Special Education Graduate Studies Program. He is also currently the General Director of ASPACE Cantabria, Santander, and a Founding Member and President of CERMi Cantabria. He is a coauthor of Síndromes y Apoyos. Panorámica Desde la Ciencia y Desde las Asociaciones (FEAPS, 2006).

Teresa González Franco received the M.Sc. degree in psychology.

She is currently a Deputy General Director with ASPACE Cantabria, Santander, Spain. She gives lectures at the Graduate Studies Program in Ed.S. degree in Special Education in the module "Education of children with cerebral palsy." She is a coauthor of Atención Educativa a alumnos con Parálisis Cerebral, edited by Confederación ASPACE and Guía de Estándares de Calidad en Atención Temprana (IMSERSO, 2004), later translated and reedited in English.

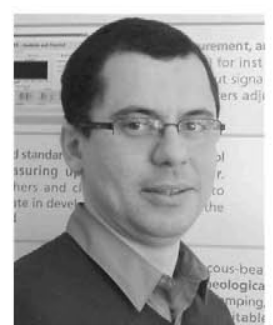

Eduardo Rocon was born in Vitoria, Brazil, in 1979. He received the Electrical Engineering degree from Universidade Federal do Espiríto Santo (UFES), Vitória, Brazil, in 2001 and the Ph.D. degree in industrial engineering from the Universidad Politécnica de Madrid, Madrid, Spain, with Prof. A. Barrientos and Prof. J. L. Pons, in 2006.

From 1999 to 2000 , he was a Research Associate with Laboratório de Automação Inteligente and successfully held a CNPq scholarship at UFES. He continued his work in tremor suppression and the application of neuroprosthetics and neurorobotics in rehabilitation on a postdoctoral contract from 2006 to 2009. Since 2010, he has been a Researcher with the Bioengineering Group, Spanish National Council for Science Research, Madrid, Spain. His multidisciplinary work has contributed to different aspects of robotics, neuroscience, and medicine. His research activities have generated more than 40 publications in indexed journals, one book, nine book chapters, more than 50 articles in international conferences, 50 articles in national conferences, five articles in journal of scientific diffusion, and seven patents.

Dr. Rocon received a Ramón y Cajal Contract, the most competitive and prestigious postdoctoral contract in Spain in 2009; the Juan Lopez de Peñalver Award of the Spanish Royal Academy of Engineering; and the Georges Giralt Ph.D. Award for his Ph.D. thesis, which focused on the development of a rehabilitation robotic exoskeleton that provides a means of testing and validating nongrounded control strategies for robotic exoskeletons for active upper limb tremor suppression. 